\title{
Y-graft modification to the Fontan procedure: Increasingly balanced flow over time
}

\author{
Phillip M. Trusty, PhD, ${ }^{\mathrm{a}}$ Zhenglun Wei, PhD, ${ }^{\mathrm{a}}$ Megan Sales, BS, ${ }^{\mathrm{b}}$ Kirk R. Kanter, MD, ${ }^{\mathrm{c}}$ \\ Mark A. Fogel, MD, ${ }^{\mathrm{d}}$ Ajit P. Yoganathan, PhD, ${ }^{\mathrm{a}}$ and Timothy C. Slesnick, MD
}

\section{ABSTRACT}

Objective: The use of Y-grafts for Fontan completion is hypothesized to offer more balanced hepatic flow distribution (HFD) and decreased energy losses. The purpose of this study was to evaluate the hemodynamic performance of Y-grafts over time using serial cardiac magnetic resonance data and to compare their performance with extracardiac Fontan connections.

Methods: Ten Fontan patients with commercially available Y-graft connections and serial postoperative cardiac magnetic resonance data were included in this study. Patient-specific computational fluid dynamics simulations were used to estimate HFD and energy losses. Y-graft performance was compared with 3 extracardiac conduit Fontan groups $(\mathrm{n}=10$ for each) whose follow-up times straddle the Y-graft time points.

Results: Y-graft HFD became significantly more balanced over time (deviation from $50 \%$ decreased from $18 \% \pm 14 \%$ to $8 \% \pm 8 \% ; P=.015)$. Total cavopulmonary connection resistance did not significantly change. Y-grafts at 3-year follow-up showed more balanced HFD than the extracardiac conduit groups at both the earlier and later follow-up times. Total cavopulmonary connection resistance was not significantly different between any Y-graft or extracardiac conduit group.

Conclusions: Y-grafts showed significantly more balanced HFD over a 3-year follow-up without an increase in total cavopulmonary connection resistance, and therefore may be a valuable option for Fontan completion. Additional follow-up data at longer follow-up times are still needed to thoroughly characterize the potential advantages of Y-graft use. ( $\mathrm{J}$ Thorac Cardiovasc Surg 2020;159:652-61)

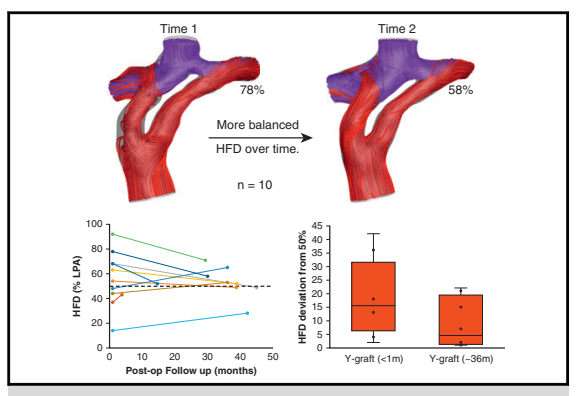

Improved Fontan Y-graft performance as follow-up time increases.

\section{Central Message}

Y-graft hepatic flow distribution significantly improves over time, offering a potential advantage over extracardiac conduit options.

\section{Perspective}

Y-graft hepatic flow distribution became significantly more balanced over an approximately 3-year follow-up with comparable TCPC resistance to extracardiac conduit options. Therefore, Y-grafts may be a valuable option for Fontan completion with inherent advantages in hepatic flow distribution.

See Commentaries on pages 662 and 665.
From the ${ }^{\mathrm{a}}$ Wallace H. Coulter Department of Biomedical Engineering, Georgia Insti-

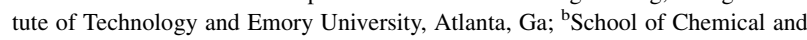
Biomolecular Engineering, Georgia Institute of Technology, Atlanta, Ga; Divisions of 'Cardiothoracic Surgery and ${ }^{\mathrm{e}}$ Cardiology, Children's Healthcare of Atlanta, Emory University School of Medicine, Atlanta, Ga; and ${ }^{\mathrm{d}}$ Division of Cardiology, Children's Hospital of Philadelphia, Philadelphia, Pa.

Supported in part by American Heart Association Predoctoral Fellowship 17PRE33630117. ANSYS software was provided through an academic partnership between ANSYS Inc and the Cardiovascular Fluid Mechanics Lab at the Georgia Institute of Technology.

Received for publication Dec 27, 2018; revisions received June 3, 2019; accepted for publication June 13, 2019; available ahead of print Aug 6, 2019

Address for reprints: Ajit P. Yoganathan, PhD, Wallace H. Coulter Department of Biomedical Engineering, Georgia Institute of Technology and Emory University, 387 Technology Cir, NW, Suite 200, Atlanta, GA 30313 (E-mail: ajit. yoganathan@bme.gatech.edu).

$0022-5223 / \$ 36.00$

Copyright $($ c 2019 by The American Association for Thoracic Surgery

https://doi.org/10.1016/j.jtcvs.2019.06.063
First performed in 1971, the Fontan procedure is the current palliation of choice for most patients with single ventricle congenital heart defects. ${ }^{1}$ In this procedure, the inferior vena cava (IVC) is anastomosed to the pulmonary arteries (PAs), typically via a conduit/graft, bypassing the subpulmonary ventricle. This reconfiguration separates oxygenated and deoxygenated blood, but creates a 1-pump

- Scanning this $\mathrm{QR}$ code will take you to the article title page to access supplementary information. 

Abbreviations and Acronyms
$\mathrm{CFD}=$ computational fluid dynamics
$\mathrm{CMR}=$ cardiac magnetic resonance
$\mathrm{ECC}=$ extracardiac conduit
HFD = hepatic flow distribution
IVC = inferior vena cava
LPA = left pulmonary artery
PA = pulmonary artery
PAVM $=$ pulmonary arteriovenous malformation
PFD = pulmonary flow distribution
$\mathrm{Q}_{\mathrm{s}} \quad=$ system venous return
$\mathrm{RPA}=$ right pulmonary artery
TCPC $=$ total cavopulmonary connection

circulatory system that requires passive blood flow through the pulmonary circulation.

In this physiology, the single ventricle must provide all cardiac power for the entire circulatory system in the presence of an added resistance due to the total cavopulmonary connection (TCPC). ${ }^{2}$ Efforts to minimize energy loss in the TCPC are hypothesized to result in improved patient outcomes and may benefit the inherently overburdened single ventricle. Additionally, due to the direct connection of the IVC to the PAs, hepatic flow has a limited opportunity to mix with upper body flow, which can result in unbalanced hepatic flow distribution (HFD) to the left and right lungs, potentially leading to the formation of pulmonary arteriovenous malformations (PAVMs). ${ }^{3-6}$ There is currently a limited understanding of the composition and production of this hepatic factor as well as the exact amount needed to preserve vascular structure. However, a balanced HFD distribution is an important factor to prevent PAVM progression. Both energy loss and HFD distribution have been the focus of numerous previous studies. $^{7-11}$

The Fontan procedure has evolved over time, progressing through the atriopulmonary, intra-atrial, and extracardiac options, with improvements in outcomes. More recently, the bifurcated Y-graft option has been selectively implemented and is proposed to alleviate the above-mentioned concerns by offering lower energy losses by avoiding caval flow collisions and encouraging more balanced HFD by directing hepatic flow through 2 Y-graft branches toward the left pulmonary artery (LPA) and right pulmonary artery (RPA). ${ }^{8,10,12-15}$

However, these benefits have not yet been fully realized. In our previous study comparing the hemodynamic performance of commercially available Y-grafts with the more traditional lateral tunnel and extracardiac conduit (ECC) options, no definite advantages were seen in patients

TABLE 1. Clinical and hemodynamic data for the extracardiac conduit (ECC) comparison groups

\begin{tabular}{|c|c|c|c|}
\hline & $\begin{array}{c}\text { ECC group 1 } \\
(\text { follow-up }<3 \text { y) }(n=10)\end{array}$ & $\begin{array}{c}\text { ECC group 2 } \\
(\text { follow-up 3-6 y) }(n=10)\end{array}$ & $\begin{array}{c}\text { ECC group 3 } \\
(\text { follow-up }>10 \text { y) }(n=10)\end{array}$ \\
\hline Age (y) & $3.30 \pm 0.95$ & $6.90 \pm 1.0$ & $16.3 \pm 3.33$ \\
\hline $\operatorname{BSA}\left(\mathrm{m}^{2}\right)$ & $0.63 \pm 0.06$ & $0.86 \pm 0.18$ & $1.48 \pm 0.28$ \\
\hline Follow-up time (y) & $0.67 \pm 0.55$ & $5.0 \pm 0.63$ & $13.5 \pm 1.78$ \\
\hline Male gender & 4 & 6 & 3 \\
\hline HLHS morphology & 5 & 6 & 5 \\
\hline Fenestrated & 7 & 7 & 6 \\
\hline Cardiac index $\left(\mathrm{L} / \mathrm{min} / \mathrm{m}^{2}\right)$ & $4.46 \pm 1.16$ & $3.66 \pm 0.86$ & $3.43 \pm 0.58$ \\
\hline IVC flow $\left(\mathrm{L} / \mathrm{min} / \mathrm{m}^{2}\right)$ & $1.08 \pm 0.32$ & $1.79 \pm 0.79$ & $1.72 \pm 0.54$ \\
\hline SVC flow $\left(\mathrm{L} / \mathrm{min} / \mathrm{m}^{2}\right)$ & $1.84 \pm 0.81$ & $1.43 \pm 0.63$ & $0.75 \pm 0.26$ \\
\hline LPA flow $\left(\mathrm{L} / \mathrm{min} / \mathrm{m}^{2}\right)$ & $1.27 \pm 0.64$ & $1.09 \pm 0.42$ & $1.07 \pm 0.35$ \\
\hline RPA flow $\left(\mathrm{L} / \mathrm{min} / \mathrm{m}^{2}\right)$ & $1.21 \pm 0.51$ & $1.78 \pm 0.89$ & $1.33 \pm 0.43$ \\
\hline $\mathrm{Q}_{\mathrm{s}}\left(\mathrm{L} / \mathrm{min} / \mathrm{m}^{2}\right)$ & $3.06 \pm 0.86$ & $2.90 \pm 0.67$ & $2.46 \pm 0.50$ \\
\hline Collateral flow $\left(\mathrm{L} / \mathrm{min} / \mathrm{m}^{2}\right)$ & $1.94 \pm 1.40$ & $0.82 \pm 0.98$ & $0.96 \pm 0.76$ \\
\hline PFD deviation (percentage points) & $13 \pm 9$ & $11 \pm 9$ & $9 \pm 7$ \\
\hline LPA stenosis (\%) & $35 \pm 24$ & $49 \pm 19$ & $32 \pm 20$ \\
\hline RPA stenosis (\%) & $20(9-31)$ & $35(10-58)$ & $19(8-32)$ \\
\hline Overall PA stenosis $(\%)$ & $27 \pm 17$ & $40 \pm 13$ & $26 \pm 15$ \\
\hline TCPC resistance (WU) & $0.29 \pm 0.37$ & $0.12 \pm 0.07$ & $0.21 \pm 0.15$ \\
\hline HFD deviation (percentage points) & $24 \pm 15$ & $21 \pm 18$ & $12 \pm 5$ \\
\hline
\end{tabular}

Values are presented as mean \pm standard deviation for normally distributed variables, median (interquartile range) for nonparametric data, or as number. ECC, Extracardiac conduit; $B S A$, body surface area; $H L H S$, hypoplastic left heart syndrome; $I V C$, inferior vena cava $S V C$, superior vena cava; $L P A$, left pulmonary artery $R P A$, right pulmonary artery; $Q_{s}$, systemic return; $P F D$, pulmonary flow distribution; $P A$, pulmonary artery; $T C P C$, total cavopulmonary connection; $W U$, Wood unit; $H F D$, hepatic flow distribution. 
with Y-grafts. ${ }^{8}$ Overall, HFD was not statistically different between graft types and TCPC resistance was higher in the Y-graft cohort. Furthermore, extremely unbalanced HFD was seen more often in patients with Y-grafts. A key limitation noted in that previous study was the difference in follow-up times between the Y-graft $(<1$ month) and lateral tunnel/ECC ( $\sim 9$ months) patients. In a study by Yang and colleagues, ${ }^{14}$ HFD was highly variable among Y-graft patients in the early postoperative state, and remained varied at a 6-month follow-up, but included a small number of patients $(\mathrm{n}=3) .{ }^{14}$ These authors illustrate that in the immediate postoperative setting the theoretical advantages of the Y-graft have not always been seen, but the anecdotal experience in our institution has been favorable. No larger series exist evaluating the midterm outcomes of patients receiving Y-graft Fontans to help advise future practice.

Motivated by these previous observations regarding this new group of Fontan patients, we sought to evaluate how the hemodynamic performance of Y-grafts changes over time. In this study, serial cardiac magnetic resonance (CMR) data and computational fluid dynamics (CFD) were used to investigate these changes. Finally, Y-graft performance was compared with ECC patients.

\section{METHODS \\ Patient Selection}

Ten Fontan patients with Y-graft connections were included in this study. Inclusion criteria included single ventricle patient with Y-graft Fontan connection; serial, postoperative CMR and phase-contrast CMR data; and sufficient image quality to accurately segment the TCPC and blood flow waveforms at each TCPC inlet/outlet throughout the cardiac cycle. All Y-graft patient data were received from Children's Healthcare of Atlanta (institutional review board No. H09279) and are numbered chronologically in order of surgery date. All Y-graft connections were implanted using commercially available aortoiliac polytetrafluoroethylene bifurcated grafts (Gore, Flagstaff, Ariz) without further modification. An ECC comparison group with identical follow-up times was not available. Therefore, to put the Y-graft results in context with ECC patients, several unique ECC groups were chosen (each $n=10$ ) with follow-up times both less than and greater than the Y-graft cohort. Each ECC group included consecutive patients within a predefined follow-up window as specified in Table 1. All ECC patient data were received from Children's Hospital of Philadelphia (institutional review board No. H05236). A sensitivity analysis was performed to ensure these small comparison groups were representative of larger Fontan cohorts. Clinical data, including age, body surface area, gender, ventricle morphology, presence of fenestration and dates of surgery, and CMR studies were obtained for each patient.

\section{CMR Acquisition}

All CMRs were performed with a Siemens 1.5 T magnetic resonance imaging system (Siemens Medical Solutions, Malvern, Pa). Patients were scanned supine, head first in the scanner, with echocardiogram leads

TABLE 2. Clinical and hemodynamic data for the Y-graft cohort

\begin{tabular}{|c|c|c|c|}
\hline & \multicolumn{2}{|c|}{ Y-graft $(n=10)$} & \multirow{2}{*}{$\frac{\text { Paired sample } t \text { test }}{P \text { value }}$} \\
\hline & Time 1 & Time 2 & \\
\hline Age (y) & $3.26 \pm 1.02$ & $5.65 \pm 1.25$ & - \\
\hline $\operatorname{BSA}\left(\mathrm{m}^{2}\right)$ & $0.65 \pm 0.10$ & $0.86 \pm 0.12$ & - \\
\hline Follow-up time (y) & $0.04(0.02-0.07)$ & $3.02(2.13-3.31)$ & $<.001 *$ \\
\hline Male gender & \multicolumn{2}{|c|}{4} & - \\
\hline HLHS morphology & \multicolumn{2}{|c|}{3} & - \\
\hline Fenestrated & \multicolumn{2}{|c|}{9} & - \\
\hline Cardiac index $\left(\mathrm{L} / \mathrm{min} / \mathrm{m}^{2}\right)$ & $3.33 \pm 0.91$ & $3.32 \pm 0.93$ & .982 \\
\hline IVC flow $\left(\mathrm{L} / \mathrm{min} / \mathrm{m}^{2}\right)$ & $0.61 \pm 0.23$ & $1.03 \pm 0.25$ & $.005^{*}$ \\
\hline $\mathrm{SVC}$ flow $\left(\mathrm{L} / \mathrm{min} / \mathrm{m}^{2}\right)$ & $1.19 \pm 0.28$ & $1.38 \pm 0.33$ & .224 \\
\hline LPA flow $\left(\mathrm{L} / \mathrm{min} / \mathrm{m}^{2}\right)$ & $0.88 \pm 0.33$ & $0.98 \pm 0.31$ & .374 \\
\hline RPA flow $\left(\mathrm{L} / \mathrm{min} / \mathrm{m}^{2}\right)$ & $0.88 \pm 0.38$ & $1.04 \pm 0.39$ & .200 \\
\hline $\mathrm{Q}_{\mathrm{s}}\left(\mathrm{L} / \mathrm{min} / \mathrm{m}^{2}\right)$ & $1.80 \pm 0.34$ & $2.40 \pm 0.53$ & $.020^{*}$ \\
\hline Collateral flow $\left(\mathrm{L} / \mathrm{min} / \mathrm{m}^{2}\right)$ & $1.53 \pm 1.03$ & $0.93 \pm 0.98$ & .264 \\
\hline PFD deviation (percentage points) & $13 \pm 11$ & $9 \pm 8$ & .173 \\
\hline LPA stenosis $(\%)$ & $17 \pm 10$ & $17 \pm 13$ & .987 \\
\hline RPA stenosis (\%) & $13(4-29)$ & $13(5-32)$ & .760 \\
\hline Overall PA stenosis (\%) & $19 \pm 13$ & $18 \pm 12$ & .847 \\
\hline TCPC resistance (WU) & $0.30 \pm 0.21$ & $0.17 \pm 0.07$ & .117 \\
\hline HFD deviation (percentage points) & $18 \pm 14$ & $8 \pm 8$ & $.015^{*}$ \\
\hline
\end{tabular}

Values are presented as mean \pm standard deviation for normally distributed variables, median (interquartile range) for nonparametric data, or as number. $B S A$, Body surface area; $H L H S$, hypoplastic left heart syndrome; IVC, inferior vena cava; $S V C$, superior vena cava; $L P A$, left pulmonary artery; $R P A$, right pulmonary artery; $Q_{s}$, systemic return; $P F D$, pulmonary flow distribution; $P A$, pulmonary artery; $T C P C$, total cavopulmonary connection; WU, Wood units; $H F D$, hepatic flow distribution. *Statistically significant. 


\begin{tabular}{|l|c|c|c|c|c|c|c|c|c|c|}
\hline & $\begin{array}{c}\mathrm{Y} 1 \\
(42 \mathrm{~m})\end{array}$ & $\begin{array}{c}\mathrm{Y} 2 \\
(39 \mathrm{~m})\end{array}$ & $\begin{array}{c}\mathrm{Y} 3 \\
(45 \mathrm{~m})\end{array}$ & $\begin{array}{c}\mathrm{Y} 4 \\
(39 \mathrm{~m})\end{array}$ & $\begin{array}{c}\mathrm{Y} 5 \\
(36 \mathrm{~m})\end{array}$ & $\begin{array}{c}\mathrm{Y} 6 \\
(29 \mathrm{~m})\end{array}$ & $\begin{array}{c}\mathrm{Y} 7 \\
(15 \mathrm{~m})\end{array}$ & $\begin{array}{c}\mathrm{Y} 8 \\
(4 \mathrm{~m})\end{array}$ & $\begin{array}{c}\mathrm{Y} 9 \\
(36 \mathrm{~m})\end{array}$ & $\begin{array}{c}\mathrm{Y} 10 \\
(30 \mathrm{~m})\end{array}$ \\
\hline HFD T1 & 14 & 54 & 68 & 63 & 48 & 92 & 68 & 37 & 44 & 78 \\
\hline HFD T2 & 28 & 49 & 49 & 52 & 65 & 71 & 52 & 43 & 53 & 58 \\
\hline
\end{tabular}

A
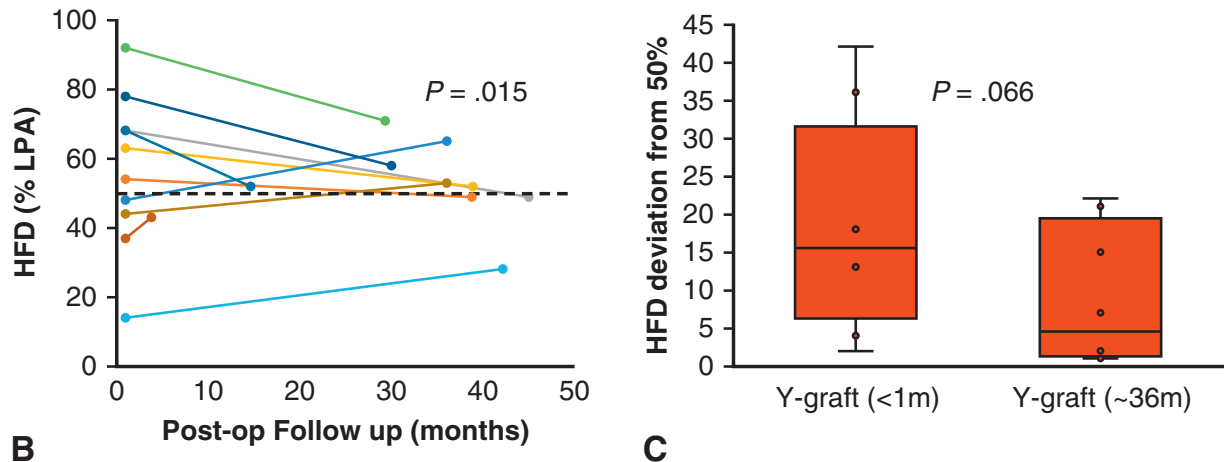

FIGURE 1. Serial comparison of Y-graft hepatic flow distribution (HFD). A, Individual patient HFD values at time 1 (T1) and time 2 (T2). B, Change in HFD over time. Each line represents a single patient. $P$ value indicates significance of paired sample $t$ test. C, Overall comparison of HFD deviation for the entire cohort. $P$ value indicates significance of independent sample $t$ test. Follow up time is indicated as number of months. Dashed black line represents ideal HFD. LPA, Left pulmonary artery.

placed. After localizers were obtained, a stack of contiguous, static, diastolic steady state free precession images were obtained from the diaphragm to thoracic inlet to assess anatomy. Slice thickness was generally 1 to $3 \mathrm{~mm}$ and in plane resolution was $1 \times 1 \mathrm{~mm}$.

Retrospectively gated, through plane phase contrast magnetic resonance was used to assess flows in the vena cavae, branch pulmonary arteries, and across the aortic valve. Inferior vena cava flow was measured suprahepatic. Velocity encoding was generally $150 \mathrm{~cm} / \mathrm{s}$ for the aorta and $60 \mathrm{~cm} / \mathrm{s}$ for the other vessels. Slice thickness was generally $4 \mathrm{~mm}$ with in plane resolution of $1 \times 1 \mathrm{~mm}$. The number of phases was a function of the heart rate and ranged from 20 to 30 .

\section{Anatomic Reconstruction and Blood Flow Segmentation}

Patient-specific anatomies were reconstructed from axial CMR images using methods previously developed. ${ }^{16,17}$ Geomagic Studio (Geomagic Inc, Research Triangle Park, NC) was used to fit a surface around the reconstructed point cloud and export the surface for mesh generation. Patient-specific blood flow waveforms were segmented from phase contrast magnetic resonance images for all vessels of interest using previously validated methods. ${ }^{18,19}$

\section{CFD}

The 3-dimensional anatomy data were imported into ANSYS workbench (Ansys Inc, Canonberg, Pa), where vessel extensions of length 10 $\times$ (vessel diameter, $\mathrm{mm}$ ) were added to overcome entrance effects and establish an appropriate velocity profile. A polyhedral mesh of approximately $\mathrm{D}_{\text {avg }} / 30 \mathrm{~mm}$ elements was used to achieve mesh independent results, where $\mathrm{D}_{\text {avg }}$ is the average vessel diameter. ${ }^{20}$ All simulations were performed using ANSYS Fluent (Release 17.0), which is a finite volume pressure based Navier-Stokes solver. Blood was modeled as a singlephase Newtonian fluid $\left(\mu=0.04 \mathrm{~g} /(\mathrm{cm} \cdot \mathrm{s}), \rho=1.06 \mathrm{~g} / \mathrm{cm}^{3}\right)$ and rigid walls were assumed. The appropriate patient-specific blood flow waveforms extracted from phase contrast magnetic resonance imaging were used as boundary conditions for each TCPC inlet and outlet. Twenty cardiac cycles were simulated to overcome transition effects and achieve period stability, using the final cycle for data analysis.

\section{Data Analysis}

HFD and TCPC resistance were assessed using CFD. HFD was quantified by seeding massless particles at the IVC and calculating the total flux of particles leaving the LPA and right PA (RPA). HFD is defined as $H F D=\frac{\theta_{L P A}}{\theta_{L P A}+\theta_{R P A}}$ where $\theta$ is the total flux of particles throughout a cardiac cycle. HFD is discussed in terms of HFD deviation from $50 \%$, and an even (50/50) split of hepatic flow to the LPA and RPA is assumed as ideal. This convention gives a 0 HFD deviation for balanced HFD cases, and an HFD deviation of 50 for extreme cases (all hepatic flow to either the LPA and RPA). Pulmonary flow distribution (PFD) is calculated as $P F D=\frac{Q_{L P A}}{Q_{L P A}+Q_{R P A}}$ where $\mathrm{Q}$ is flow rate. Collateral flow was calculated as the difference between aortic and vena cava flow. TCPC resistance is calculated as TCPC Resistance $=\left(\frac{\Delta P_{T C P C}}{Q_{s}}\right)$ where $\triangle P_{T C P C}$ is the pressure drop across the TCPC (calculated via CFD) and $\mathrm{Q}_{\mathrm{s}}$ is the systemic venous flow. Flow rates are indexed to body surface area.

Percent stenosis was calculated for the PAs using the following equation:

$$
\text { Stenosis } \%=100\left(\frac{A_{\text {min }, L P A}+A_{\text {min }, R P A}}{A_{\text {avg }, L P A}+A_{\text {avg }, R P A}}\right)
$$

where $A_{m i n, L P A}$ and $A_{m i n, R P A}$ are the minimum cross-sectional areas of the LPA and RPA, and $A_{\text {avg, LPA }}$ and $A_{\text {avg, RPA }}$ are the average crosssectional areas of the LPA and RPA. A combined outlet stenosis value was used in place of unilateral PA stenosis measurements to give a more accurate representation of the total outlet obstruction to flow and allow a better comparison between patients. Additionally, IVCs and superior vena cavas showed negligible percent stenosis and therefore were not included in this calculation. All vessel areas for this metric were calculated using Vascular Modeling Toolkit version 1.0.1 (Orobix, Bergamo, Italy). 


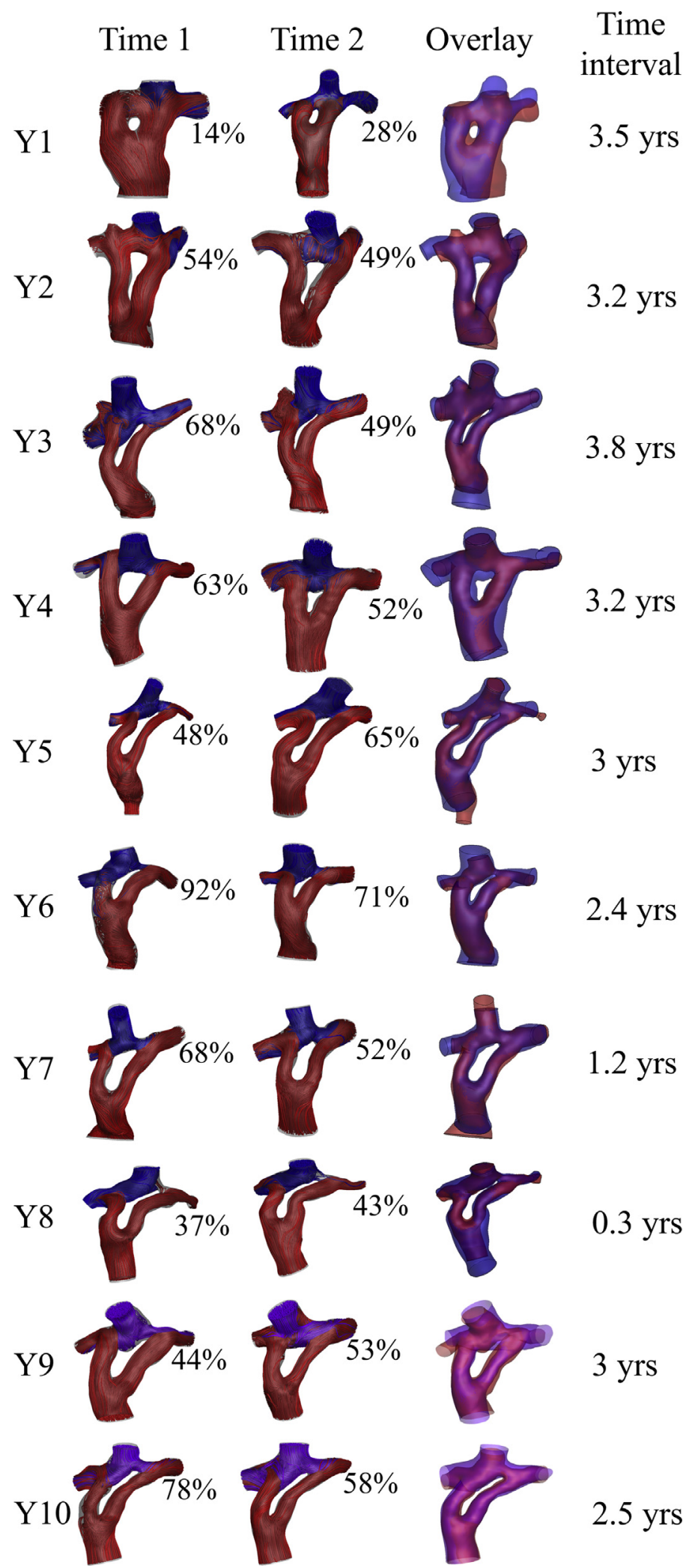

FIGURE 2. Streamlines for all Y-graft patients. The overlay image compares anatomies at time 1 (red) and time 2 (blue). Time interval indicates time between time 1 and time 2. Hepatic flow distribution (HFD) is indicated by percent of HFD to the left pulmonary artery. 


\begin{tabular}{|l|c|c|c|c|c|c|c|c|c|c|}
\hline & $\begin{array}{c}\mathrm{Y} 1 \\
(42 \mathrm{~m})\end{array}$ & $\begin{array}{c}\mathrm{Y} 2 \\
(39 \mathrm{~m})\end{array}$ & $\begin{array}{c}\mathrm{Y} 3 \\
(45 \mathrm{~m})\end{array}$ & $\begin{array}{c}\mathrm{Y} 4 \\
(39 \mathrm{~m})\end{array}$ & $\begin{array}{c}\mathrm{Y} 5 \\
(36 \mathrm{~m})\end{array}$ & $\begin{array}{c}\mathrm{Y} 6 \\
(29 \mathrm{~m})\end{array}$ & $\begin{array}{c}\mathrm{Y} 7 \\
(15 \mathrm{~m})\end{array}$ & $\begin{array}{c}\mathrm{Y} 8 \\
(4 \mathrm{~m})\end{array}$ & $\begin{array}{c}\mathrm{Y9} \\
(36 \mathrm{~m})\end{array}$ & $\begin{array}{c}\mathrm{Y} 10 \\
(30 \mathrm{~m})\end{array}$ \\
\hline $\mathrm{R}_{\mathrm{TCPC}} \mathrm{T} 1$ & 0.06 & 0.13 & 0.22 & 0.70 & 0.60 & 0.18 & 0.31 & 0.34 & 0.35 & 0.12 \\
\hline $\mathrm{R}_{\mathrm{TCPC}} \mathrm{T} 2$ & 0.33 & 0.15 & 0.14 & 0.11 & 0.18 & 0.13 & 0.14 & 0.23 & 0.17 & 0.11 \\
\hline
\end{tabular}

A
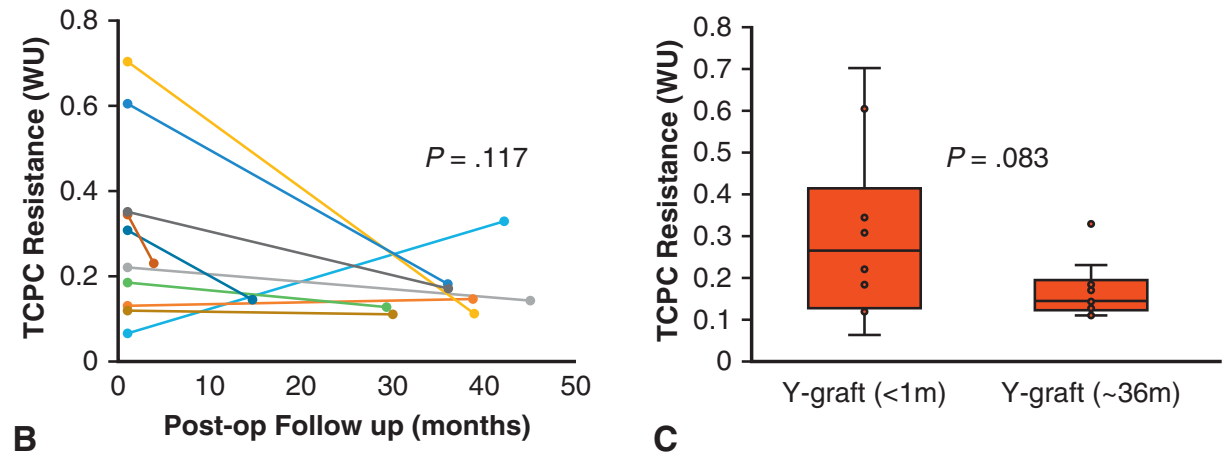

C

FIGURE 3. Serial comparison of Y-graft total cavopulmonary connection resistance $\left(R_{T C P C}\right)$. A, Individual patient $\mathrm{R}_{\mathrm{TCPC}}$ values at time 1 (T1) and time 2 (T2). B, Change in $\mathrm{R}_{\mathrm{TCPC}}$ over time. Each line represents a single patient. $P$ value indicates significance of paired sample $t$ test. C, Overall comparison of $\mathrm{R}_{\mathrm{TCPC}}$ for entire cohort. $P$ value indicates significance of independent sample $t$ test. $W U$, Wood units.

\section{Statistical Analysis}

SPSS version 25, (IBM-SPSS Inc, Armonk, NY) was used for statistical analyses. The Shapiro-Wilk test was used to determine normality for each parameter. Pearsos and Spearman correlations were used to investigate bivariate correlations between TCPC resistance and HFD with clinical, hemodynamic, and anatomical parameters. Paired sample $t$ tests or Wilcoxon signed-rank tests were used to test for significant changes over time in the Y-graft cohort for parametric and nonparametric data, respectively. Independent sample $t$ tests and Wilcoxon sum rank tests were used to compare means between the Y-graft and ECC comparison groups for parametric and nonparametric data, respectively. Statistical significance was determined at the $P=.05$ level. Values are presented as average \pm standard deviation for normally distributed variables and median (interquartile range [IQR]) for nonparametric data.

\section{RESULTS}

\section{Y-Grafts}

Clinical and hemodynamic data for all Y-grafts at both time points are shown in Table 2. Age increased from $3.26 \pm 1.02$ years to $5.65 \pm 1.25$ years across the 2 time points, with an average follow-up time of 14 days (IQR, 7-25 days) and 3.02 years (IQR, 2.13-3.31 years),
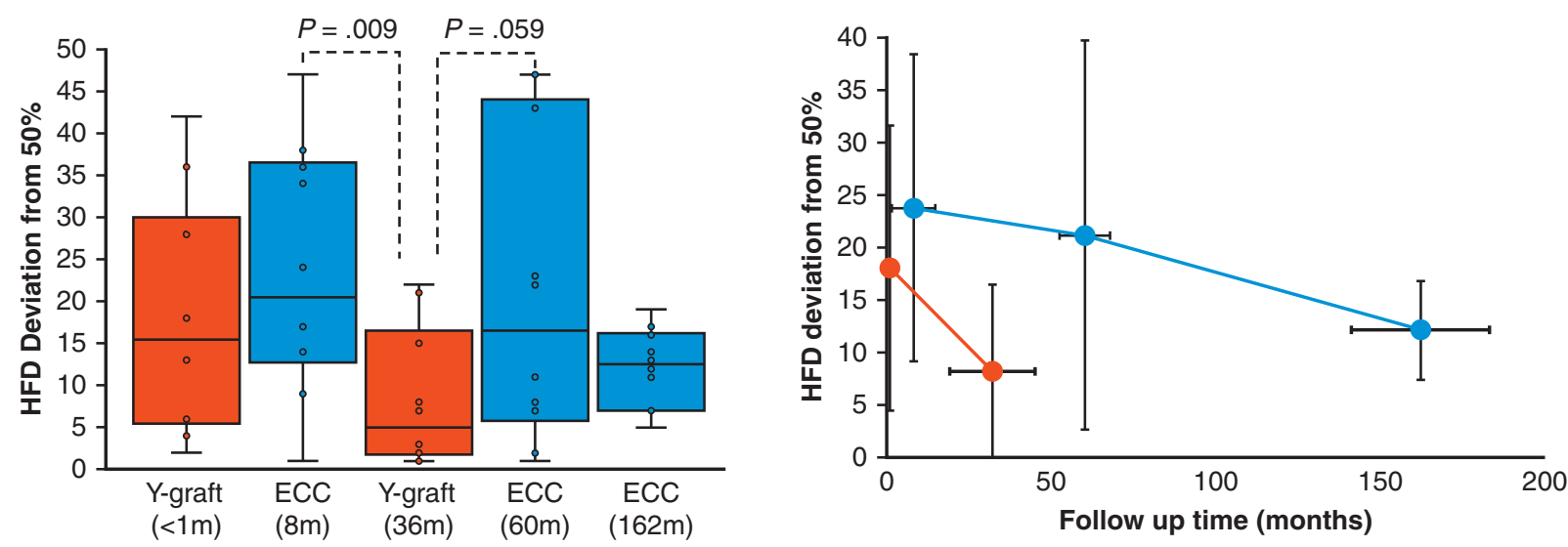

A

B

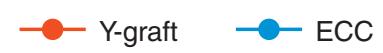

FIGURE 4. Hepatic flow distribution (HFD) comparison of Y-grafts and extracardiac conduit (ECC) patients. A, Box plot of results. B, line graph of results. For the box plot, the upper and lower borders of the box represent the upper and lower quartiles. The middle horizontal line represents the median. The upper and lower whiskers represent the maximum and minimum values of nonoutliers. Dots represent individual data points. Dots are not duplicated on the whiskers or median line. Error bars in the line graph represent standard deviation. $P$ value indicates significance of independent sample $t$ test. No other comparison was significant. 


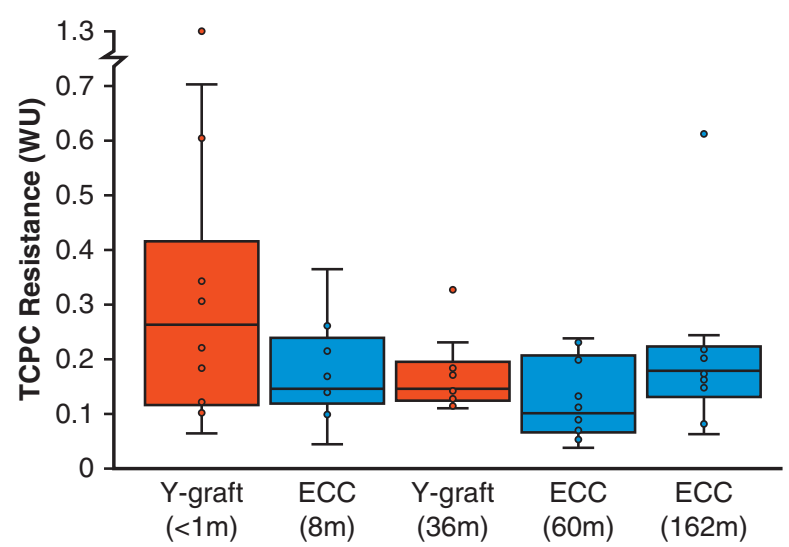

A

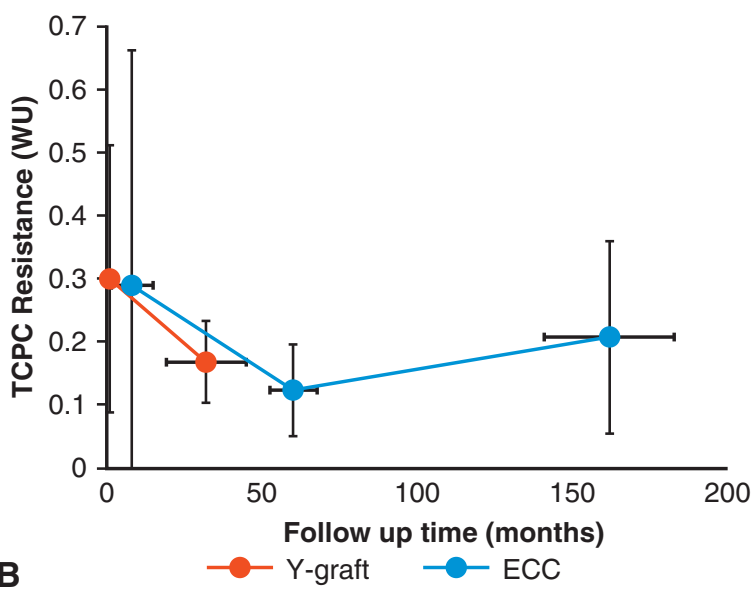

B

FIGURE 5. Total cavopulmonary connection (TCPC) resistance comparison of Y-grafts and extracardiac conduit (ECC) patients. A, Box plot of results. B, Line graph of results. For the box plot, the upper and lower borders of the box represent the upper and lower quartiles. The middle horizontal line represents the median. The upper and lower whiskers represent the maximum and minimum values of nonoutliers. Dots represent individual data points. Dots are not duplicated on the whiskers or median line. Error bars in the line graph represent standard deviation. No statistical comparison between groups was significant. $W U$, Wood units.

respectively. The cohort included 4 men and the most common underlying anatomy was hypoplastic left heart syndrome, present in 3 patients. Clinically, these Y-graft patients are doing well, with no Fontan complications (eg, protein losing enteropathy or plastic bronchitis) and oxygen saturations in the mid to upper 90s. These patients are currently aged 7 to 10 years and none are being evaluated for transplantation.

Indexed IVC flow increased from $0.57 \mathrm{~L} / \mathrm{min} / \mathrm{m}^{2}$ (IQR, $\left.0.45-0.89 \mathrm{~L} / \mathrm{min} / \mathrm{m}^{2}\right)$ to $1.03 \pm 0.25 \mathrm{~L} / \mathrm{min} / \mathrm{m}^{2}(P=.005)$. Similarly, $Q_{\mathrm{s}}$ increased from $1.80 \pm 0.34 \mathrm{~L} / \mathrm{min} / \mathrm{m}^{2}$ to $2.40 \pm 0.53 \mathrm{~L} / \mathrm{min} / \mathrm{m}^{2}(P=.020)$. Superior vena cava, LPA, RPA, collateral flow, and cardiac index showed no significant changes between time points. Pulmonary flow distribution became more balanced (PFD deviation from 50\% decreased from $13 \% \pm 11 \%$ to $9 \% \pm 8 \%$ ) although not statistically significant $(P=.173)$. Finally, PA stenosis did not significantly change over time.

\section{HFD}

HFD significantly improved over time (paired sample $t$ test, $P=.015$ ) (Table 2). HFD deviation decreased from $18 \% \pm 14 \%$ to $8 \% \pm 8 \%$. Nine out of 10 patients had more balanced HFD at time 2 than time 1 (Figure 1). On average, there was a $10 \pm 10$ point improvement in HFD deviation. Individual HFD values and follow-up times are shown in Figure 1, A. Streamlines for all Y-grafts at both timepoints are shown in Figure 2. Overall, HFD was relatively balanced at time 2 with an HFD deviation of only $8 \% \pm 8 \%$.

\section{TCPC Resistance}

TCPC resistance decreased from $0.30 \pm 0.21$ to $0.17 \pm 0.07$ wood units (WU), but was not statistically significant (paired sample $t$ test, $P=.117$ ) (Table 2). TCPC resistance decreased for 8 out of 10 patients (Figure 3). Individual resistance values can be found in Figure 3, A.

\section{Comparison With ECC Control Groups}

Three ECC comparison groups were chosen using consecutive patients with $<3$-year follow-up, 3- to 6-year follow-up, and $>10$-year follow up ( $\mathrm{n}=10$ for each group). These 3 groups were chosen to straddle the Y-graft follow-up times because a comparison group with identical, serial follow-up times was not available. The clinical and hemodynamic data for all 3 ECC comparison groups are shown in Table 1. Follow-up times were used as a selection criterion, and was therefore different for the 3 comparison groups: $0.67 \pm 0.55$ years, $5.0 \pm 0.63$ years, and $13.5 \pm 1.78$ years, respectively. All ECC patients in the 2 younger comparison groups are free from Fontan complications. Two patients in the oldest comparison group were reported to have mild protein losing enteropathy. None of these patients are being evaluated for transplantation.

Both Y-grafts and ECC patients showed more balanced HFD as patients aged (Figure 4). Y-grafts improved quicker than ECC. The Y-grafts at time 2 (36-month follow-up) were significantly more balanced than the ECC group with an earlier follow-up time $(P=.009)$, and more balanced, although not statistically significant, than the 2 ECC groups with later follow-up times $(P=.059$ and $P=.209$, respectively) (Figure $4, A$ ). TCPC resistance was not significantly different between any 2 groups, although slightly higher in the Y-graft patients at time 1 (Figure 5). Large variations in TCPC resistance were seen 


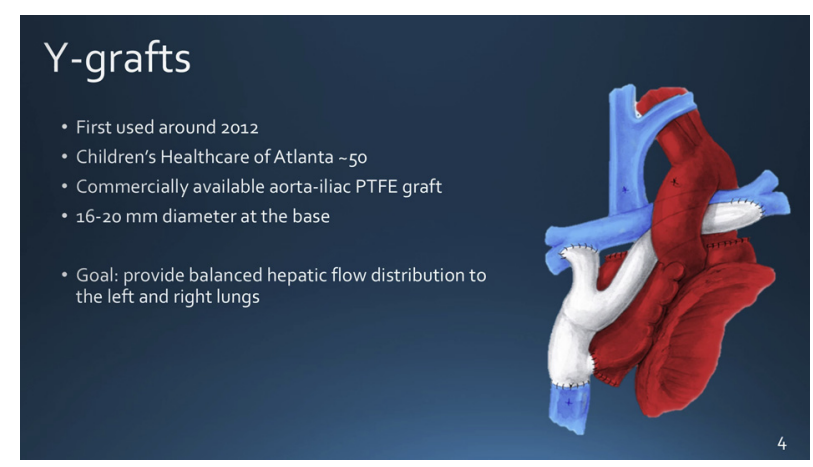

VIDEO 1. Discussion of Y-graft results. Video available at: https://www. jtcvs.org/article/S0022-5223(19)31362-5/fulltext.

at the earliest timepoints in both Y-grafts and ECC patients, and these variations decreased in the later timepoints.

\section{DISCUSSION}

The concept of using bifurcated Y-grafts for Fontan completion was initially met with interest as an intuitive solution for achieving balanced HFD and avoiding caval flow collisions. However, early evaluations failed to confirm these potential advantages. ${ }^{8}$ The operation is inherently more complex than more traditional Fontan completions, in that it involves 3 anastomotic sites rather than 2 and has caliber changes throughout the bifurcated baffle as flow moves from the main trunk into the Y-arms. Additionally, the geometry of a Y-shaped baffle lends itself to more potential for twisting and kinking than does a straight tube baffle. These limitations notwithstanding, we have not found the Y-graft Fontan to be inferior to the traditional external or later tunnel connections, simply that it was not superior in the metrics evaluated on early follow-up studies. We therefore believe its potential for streamlined flow and improved efficiency merits ongoing consideration and study (Video 1). Figure 6 highlights 1 representative patient and summarizes these findings. Although the patient numbers in this study are challenging from a statistical standpoint, it does represent by far the largest such cohort of Y-graft Fontans with midterm follow-up data.

\section{HFD}

Y-graft patients showed more balanced HFD as follow-up time increased. (Figure 4). The Y-grafts (at 36-month follow up) had a lower HFD deviation than any of the ECC groups.

In general, more balanced HFD over time (for either graft type) may be explained in part by two primary factors: more balanced overall PFD and inferior systemic venous return constituting a larger percentage of $\mathrm{Q}_{\mathrm{s}}$ as patients age. Though HFD is multifactorial, one key component is pulmonary flow distribution. In most cases, as PFD becomes more balanced, HFD becomes more balanced. In this study, both patient cohorts showed more balanced PFD as age increased (Tables 1and 2). Secondly, it is well known that the IVC receives a larger portion of $\mathrm{Q}_{\mathrm{s}}$ as patients age. ${ }^{21}$ As this percentage increases, graft positioning, flow interactions, and the location of stenosis may have a diminished effect.

In our Y-graft cohort, the early time point was obtained within the first month of the operation in all cases. Although these data are valuable and represents the patients' postoperative hemodynamic profiles, there are transient factors, such as fluid shifts, pleural effusions, and tissue edema, that were likely still resolving in many of these patients at the time of CMR. The effect of these postoperative changes on their CMR and CFD results are not entirely clear, but it is possible that some of the maldistribution of hepatic factor in these patients was only transient, and once their systems had reached a new equilibrium a more balanced state existed. For the reasons above, as children age and IVC flow increases, it is expected that HFD will become more balanced regardless of graft type. Both of these factors would be expected to occur at similar rates for the Y-graft and ECC groups, so the fact that Y-grafts exhibited an accelerated improvement in HFD when compared with the ECC group (Figure 4, $B$ ) suggests that using a Y-graft may provide an advantage over ECC connections in terms of achieving a balanced HFD. Whether the early time point underrepresented how well balanced the HFD was in Y-graft patients or whether they had more substantial normalization over time, the end result appears to be a more favorable hemodynamic state for these patients.

\section{TCPC Resistance}

Even with the built-in $50 \%$ stenosis inherent to the design of commercially available Y-grafts (the trunk diameter is double that of the individual arms, resulting in a $50 \%$ loss of cross sectional area as the blood moves through the graft), TCPC resistance was not significantly different between the Y-graft and ECC groups. Overall PA stenosis was higher in the ECC groups, but not to the extent of equaling the total (Y-graft + PA) stenosis seen in Y-graft patients. Therefore, avoiding caval flow collisions through $\mathrm{Y}$ graft use likely plays a role in decreasing energy losses and lowering TCPC resistance. This finding encourages the use of a customized Y-graft design that would preserve crosssectional area throughout the bifurcation. ${ }^{12}$ By combining a stenosis-free graft design with the ability to avoid caval flow collisions, it is reasonable to think that Y-grafts could offer lower TCPC resistance than the standard ECC connections. It remains unknown how TCPC resistance will differ between Y-grafts and ECC connections at follow-up times beyond 36 months (our longest Y-graft follow-up time). With increasing cardiac output as patients age, avoiding caval flow collisions may become a progressively more important factor. However, investigating this hypothesis requires longer term follow-up data. 

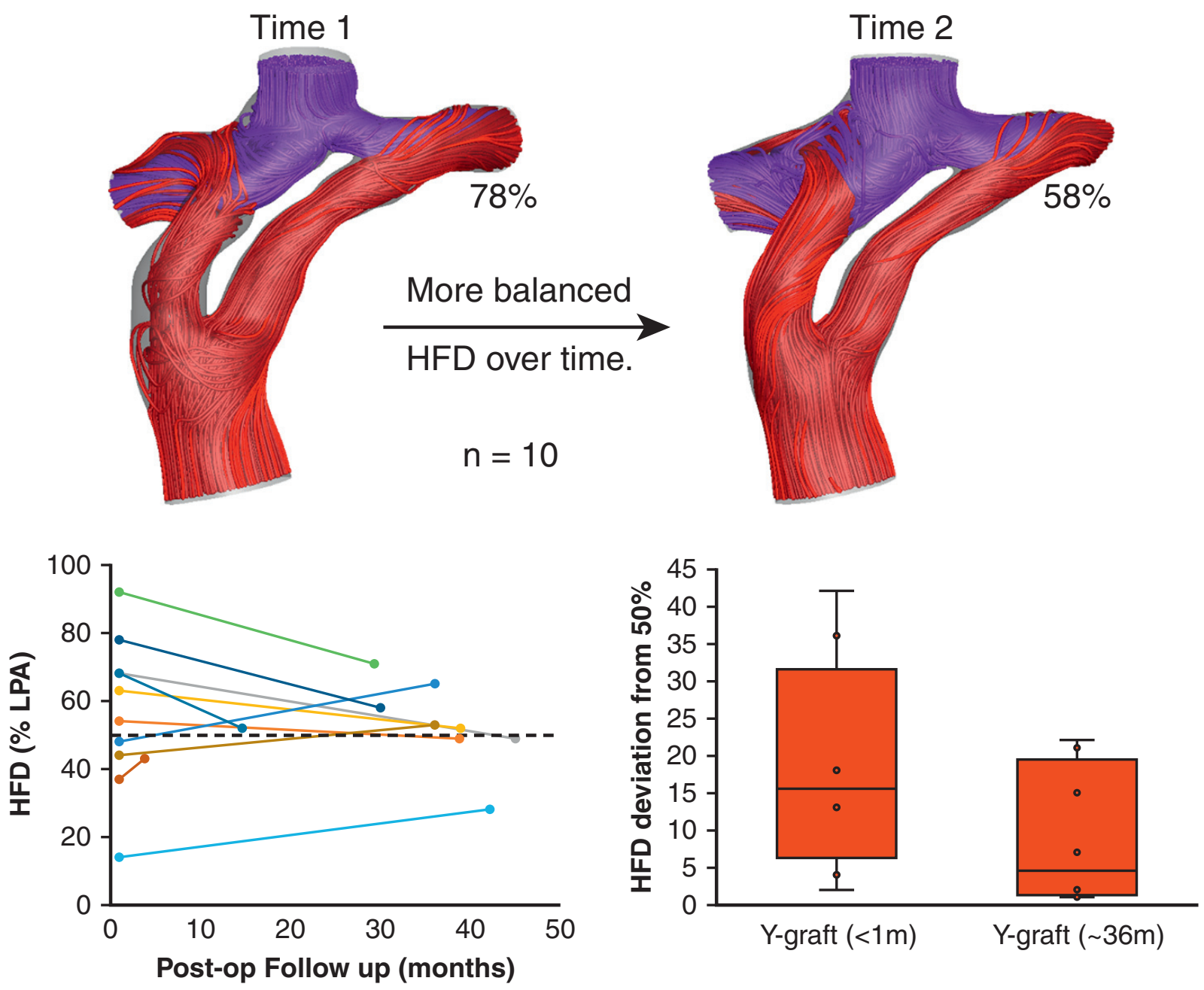

FIGURE 6. Improved Fontan Y-graft performance as follow-up time increases. Hepatic flow distribution (HFD) changes for a representative patient are shown. With an average follow-up time of approximately 3 years, Y-grafts $(n=10)$ significantly improved in terms of HFD. Nine out of 10 patients had more balanced HFD at time 2 than time 1. HFD to the left lung is indicated by percentage. LPA, Left pulmonary artery.

\section{Future Y-Graft Use}

Based on the current data, Y-graft Fontan connections are a viable surgical option. After the immediate post-operative state, Y-grafts offered more balanced HFD than ECC connections without sacrificing energy efficiency. Though longer follow up data on this unique cohort will be instructive, future Y-graft use may be a proactive step to balance HFD and reduce PAVM development. Additionally, as suggested in previous studies, the use of an area preserving Ygraft design may offer further hemodynamic benefits. ${ }^{10,12}$

\section{Limitations}

As with studies of patients with Fontan circulation, a small sample size limits the power of statistical analysis. However, even with our limited patient number a significant improvement in HFD was observed. Additionally, the lack of an ECC comparison group with identical follow-up times required the use of several unique comparison groups.
Although this does not allow for a direct comparison with serial ECC data, choosing follow-up times that straddle the Y-grafts can provide meaningful comparisons. Furthermore, although a 3-year follow-up time for this novel group of Y-graft patients is the longest to date, these new data provide limited understanding of potential long-term consequences. In addition, a 50/50 flow split to the left and right lung will not be ideal for every individual patient. This ratio was used as a consistent approximation for all patients in this study. Finally, it is important to note that all Y-graft patients in this study used commercially available Y-grafts, and results may differ with more customized designs that preserve cross-sectional area.

\section{CONCLUSIONS}

Y-grafts showed significantly more balanced HFD as follow-up time increased. Previous Y-graft concerns, including increasing resistance without improving HFD, 
may have been driven by altered hemodynamic parameters in the immediate postoperative state that did not allowing for physiologic adaptation. Additional follow-up data at increased follow-up times are needed to thoroughly characterize the potential advantages of Y-graft use.

\section{Conflict of Interest Statement}

Authors have nothing to disclose with regard to commercial support.

\section{References}

1. Fontan F, Baudet E. Surgical repair of tricuspid atresia. Thorax. 1971;26:240-8.

2. Gewillig M, Brown SC. The Fontan circulation after 45 years: update in physiology. Heart. 2016;102:1081-6.

3. Srivastava D, Preminger T, Lock JE, Mandell V, Keane JF, Mayer JE Jr, et al. Hepatic venous blood and the development of pulmonary arteriovenous malformations in congenital heart disease. Circulation. 1995;92:1217.

4. Shinohara T, Yokoyama T. Pulmonary arteriovenous malformation in patients with total cavopulmonary shunt: what role does lack of hepatic venous blood flow to the lungs play? Pediatr Cardiol. 2001;22:343-6.

5. Vettukattil JJ. Pathogenesis of pulmonary arteriovenous malformations: role of hepatopulmonary interactions. Heart. 2002;88:561-3.

6. Bernstein HS, Brook MM, Silverman NH, Bristow J. Development of pulmonary arteriovenous fistulae in children after cavopulmonary shunt. Circulation. 1995; 92:309.

7. Restrepo M, Tang E, Haggerty CM, Khiabani RH, Mirabella L, Bethel J, et al. Energetic implications of vessel growth and flow changes over time in fontan patients. Ann Thorac Surg. 2015;99:163-70.

8. Trusty PM, Restrepo M, Kanter KR, Yoganathan AP, Fogel MA, Slesnick TC. A pulsatile hemodynamic evaluation of the commercially available bifurcated Ygraft Fontan modification and comparison with the lateral tunnel and extracardiac conduits. J Thorac Cardiovasc Surg. 2016;151:1529-36.

9. Marsden AL, Vignon-Clementel IE, Chan FP, Feinstein JA, Taylor CA. Effects of exercise and respiration on hemodynamic efficiency in CFD simulations of the total cavopulmonary connection. Ann Biomed Eng. 2007;35:250-63.

10. Yang W, Feinstein JA, Shadden SC, Vignon-Clementel IE, Marsden AL. Optimization of a Y-graft design for improved hepatic flow distribution in the fontan circulation. J Biomech Eng. 2013;135:011002.
11. Sundareswaran KS, de Zélicourt D, Sharma S, Kanter KR, Spray TL, Rossignac J, et al. Correction of pulmonary arteriovenous malformation using image-based surgical planning. JACC Cardiovasc Imaging. 2009;2: 1024-30.

12. Marsden AL, Bernstein AJ, Reddy VM, Shadden SC, Spilker RL, Chan FP, et al. Evaluation of a novel Y-shaped extracardiac Fontan baffle using computational fluid dynamics. J Thorac Cardiovasc Surg. 2009;137: 394-403.e2.

13. Kanter KR, Haggerty CM, Restrepo M, et al. Preliminary clinical experience with a bifurcated Y-graft Fontan procedure - a feasibility study. J Thorac Cardiovasc Surg. 2012;144:383-9.

14. Yang W, Chan FP, Reddy VM, Marsden AL, Feinstein J. Flow simulations and validation for the first cohort of patients undergoing the Y-graft Fontan procedure. J Thorac Cardiovasc Surg. 2015;149:247-55.

15. Trusty PM, Wei Z, Tree M, Kanter KR, Fogel MA, Yoganathan AP et al. Local hemodynamic differences between commercially available Y-grafts and traditional fontan baffles under simulated exercise conditions: implications for exercise tolerance. Cardiovasc Eng Technol. 2017;8:390-9.

16. Martins T, Barbara A, Silva G, Faria T, Dalava B, Silva J. InVesalius: threedimensional medical reconstruction software. Virtual Rapid Manuf. 2007; 135-41.

17. De Moraes, Thiago F, Amorim PH. InVesalius-an open-source imaging application. Comput Vis Med Image Process. 2011;405.

18. Heiberg E, Sjögren J, Ugander M, Carlsson M, Engblom H, Arheden H. Design and validation of Segment - freely available software for cardiovascular image analysis. BMC Med Imaging. 2010;10:1.

19. Bidhult SL, Carlsson M, Steding-Ehrenborg K, Arheden H, Heiberg E. A new method for vessel segmentation based on a priori input from medical expertise in cine phase-contrast magnetic resonance imaging. J Cardiovasc Magn Reson. 2014;16(Suppl 1):P355.

20. Wei ZA, Tree M, Trusty PM, Wu W, Singh-Gryzbon S, Yoganathan A. The advantages of viscous dissipation rate over simplified power loss as a fontan hemodynamic metric. Ann Biomed Eng. 2018;46:404-16.

21. Whitehead KK, Sundareswaran KS, Parks WJ, Harris M, Yoganathan AP, Fogel M. Cardiac magnetic resonance velocity mapping study. J Thorac Cardiovasc Surg. 2010;138:96-102.

Key Words: congenital heart disease, Fontan, single ventricle, Y-graft, pulmonary arteriovenous malformations 